\title{
Toward a unified description of jet and medium scales in heavy-ion collisions
}

\author{
Konrad Tywoniuk ${ }^{* \dagger}$ \\ Theoretical Physics Department, CERN, 1211 Geneva 23, Switzerland \\ E-mail: konrad.tywoniukecern.ch
}

\begin{abstract}
Hard processes in heavy-ion collisions, in particular those involving the production of jets in the final-state, serve as well-constrained probes of a hot and dense QCD medium. At high-energies, radiation stimulated via interactions with the medium control the amount of energy radiated away from the jet constituents, providing a direct way to extract the relevant medium properties from experimental data. However, improving the precision of such comparisons has until recently been hampered by the lack of theoretical control regarding jet fragmentation inside the medium. Here, we report on first developments toward incorporating jet and medium scales on equal footing, focussing mostly on effects related to the iconic single-inclusive jet suppression factor. We demonstrate in particular how energy loss processes acts on multi-particle systems and discuss the logarithmic phase space for resummation. The progress in understanding these effects points toward a more complete description of in-medium jet fragmentation at leading-logarithmic order.
\end{abstract}

XIII Quark Confinement and the Hadron Spectrum - Confinement2018

31 July - 6 August 2018

Maynooth University, Ireland

\footnotetext{
* Speaker.

${ }^{\dagger}$ Currently at Department of Physics and Technology, University of Bergen, Allégaten 55, 5007 Bergen, Norway
} 


\section{Introduction}

The strong suppression of high- $p_{T}$ particles and jets, including heavy quarks, in heavy-ion collisions stand out as one of the hallmark measures of final-state interactions in a color deconfined medium; for an introduction, see [1]. Strikingly, the suppression of jets persists up to very large transverse momenta. This calls into question the validity of the conventional jet quenching paradigm based on single-parton energy loss because of the large available phase space for higherorder corrections. Careful considerations of the space-time evolution of jets, see [2], turns out to play an important role.

It has already been pointed out in Monte-Carlo studies that fluctuations related to the jet fragmentation, or substructure, are extremely important for understanding experimental data, see e.g. $[3,4]$, but until recently a first-principle understanding of these corrections was lacking. In the vacuum, higher-order corrections are typically not enhanced by large logarithms because of the cancellation of real and virtual contributions for sufficiently inclusive observables. In special cases, however, they are enhanced by the phase space where the virtual terms dominate, and the procedure that allows to account for such effects is generically referred to as a Sudakov resummation.

As a classic example, let us consider higher-order corrections to the singe-inclusive jet cross section in heavy-ion collisions. In contrast to the vacuum, we argue that energy loss effects that are induced by medium interactions will give rise to a mismatch between real and virtual emissions. This occurs because a real emission happening early in the medium is sensitive to the quenching of two particles along the length of the medium. In comparison, the virtual fluctuation is only affected by quenching of the parent parton because it remains unresolved by the medium. The consequences of such a mismatch can be easily illustrated by considering an extreme scenario where the medium absorbs all jet daughter particles, leaving the leading, most energetic branching unaffected. In this case, the higher-order correction is purely virtual and simply counts number of modes that are forbidden to occur inside the medium. In terms of formation times this amounts to $t_{\mathrm{f}}<L$, where $t_{\mathrm{f}} \sim 1 /\left(\omega \theta^{2}\right)$. The number of these fluctuations is then given by the phase space volume and reads $\Pi_{t_{\mathrm{f}}<L}=\frac{1}{2} \bar{\alpha} \log ^{2} p_{T} R^{2} L$ in the leading-logarithmic approximation, where $\bar{\alpha} \equiv \alpha_{S} C_{R} / \pi$. At large $p_{T}$, resumming such corrections gives rise to a jet suppression factor $R_{\text {jet }} \sim \exp \left[-\Pi_{\mathrm{t}_{\mathrm{f}}<L}\right]$ that can be significantly smaller than unity. This result however neglects color (de)coherence effects which, as we will see below, change this behavior substantially.

Our main message is that quenching modifies directly the yield of high- $p_{T}$ jets and imposes further phase space restrictions for their subsequent fragmentation, and we can work out how to deal with both in a theoretically controlled manner. Here we report on a QCD calculation [5] that resums a set of logarithmically enhanced higher-order corrections accounting for finite quenching effects. We will take particular care in defining the logarithmic phase space where large corrections occur, explaining the role of color decoherence in determining it.

\section{Higher-order corrections to jet quenching}

The jet suppression factor is defined as

$$
R_{\text {jet }}=\left(\frac{\mathrm{d} \sigma^{\mathrm{med}}}{\mathrm{d} p_{T}^{2} \mathrm{~d} y}\right) /\left(\frac{\mathrm{d} \sigma^{\mathrm{vac}}}{\mathrm{d} p_{T}^{2} \mathrm{~d} y}\right),
$$


where the $p_{T}$-spectrum in medium is modified according to [6]

$$
\frac{\mathrm{d} \sigma^{\text {med }}}{\mathrm{d} p_{T}^{2} \mathrm{~d} y}=\int_{0}^{\infty} \mathrm{d} \varepsilon \mathscr{P}(\varepsilon) \frac{\mathrm{d} \sigma^{\mathrm{vac}}\left(p_{T}+\varepsilon\right)}{\mathrm{d} p_{T}^{\prime 2} \mathrm{~d} y}
$$

where $\mathscr{P}(\varepsilon)$ is a generic probability distribution for emitting an energy $\varepsilon$ out of the jet cone. In addition to its sensitivity to the jet quenching parameter $\hat{q}$ and the medum size $L$, it also depends on the jet $p_{T}$ and cone size $R$. By approximating the steeply falling spectrum by $\mathrm{d} \sigma^{\mathrm{vac}}\left(p_{T}+\varepsilon\right) \simeq$ $\mathrm{d} \sigma^{\mathrm{vac}}\left(p_{T}\right) \mathrm{e}^{-n \varepsilon / p_{T}}$, the nuclear modification factor is simply related to the appropriate moment of the Laplace transform of the quenching weight, $R_{\text {jet }}=\mathscr{Q}\left(p_{T}\right)$, with $\mathscr{Q}\left(p_{T}\right) \equiv \tilde{\mathscr{P}}\left(p_{T} / n\right)$.

For ease of explanation, let us assume that all jets are initiated by quarks at high $p_{T}$ and expand the suppression factor $R_{\text {jet }}$ in terms of the strong coupling constant,

$$
R_{\mathrm{jet}}=\mathscr{Q}_{q}\left(p_{T}\right)+\mathscr{Q}^{(1)}\left(p_{T}\right)+\mathscr{O}\left(\alpha_{s}^{2}\right),
$$

where $\mathscr{Q}_{q}\left(p_{T}\right) \equiv \tilde{\mathscr{P}}_{q}\left(p_{T} / n\right)$ is the quenching factor of a single quark. At high- $p_{T}$, where radiative processes dominate energy loss out of the jet cone, it is computed by resumming multiple induced gluon emissions enhanced by the medium length. Technically, this allows to neglect any interference effects, and the probability distribution is found by solving the rate equation $[6,1,15]$

$$
\frac{\partial}{\partial t} \tilde{\mathscr{P}}_{q}(v, t)=\gamma(v, t) \tilde{\mathscr{P}}_{q}(v, t),
$$

up to $t=L$, where $\gamma(v, t)=\int_{0}^{\infty} \mathrm{d} \omega\left(\mathrm{e}^{-v \omega}-1\right) \mathrm{d} I /[\mathrm{d} \omega \mathrm{d} t]$ is the Laplace transform of the splitting rate (regularized by adding virtual splittings). For our present purposes, we will approximate the rate with $\mathrm{d} I /[\mathrm{d} \omega \mathrm{d} t]=\bar{\alpha} \sqrt{\hat{q} / \omega^{3}}$ that accounts for multiple, soft scattering in the medium, where $\hat{q}$ is the celebrated jet transport coefficient [7,8]. For this spectrum, $\omega_{c} \sim \hat{q} L^{2}$ acts as a cut-off energy which, if neglected, results in a time-independent rate given by,

$$
\mathscr{Q}_{q}\left(p_{T}\right)=\mathrm{e}^{-2 \bar{\alpha} L \sqrt{\pi n \hat{q} / p_{T}}} .
$$

Not surprisingly, this takes the characteristic form of a Sudakov suppression factor for the induced gluons, and the exponent is nothing but the multiplicity of gluons with $\omega>p_{T} / n$ where virtual emissions dominate over the real ones [6], see also [9] for further improvements. The regime of strong quenching, i.e. $\mathscr{Q}_{q}\left(p_{T}\right) \ll 1$, arises for $p_{T} \ll n \bar{\alpha}^{2} \omega_{c}$. We point out that since this quenching factor only knows about a single parton in the high-energy limit, there is no a priori dependence on the jet scales. Implicitly, it is however assumed that the soft radiation $\sim \bar{\alpha}^{2} \omega_{c}$ is emitted at angles much larger than the jet cone; leading to $\theta_{c} / \bar{\alpha}^{3 / 2} \gg R$, see definition below.

After this short recap, we can now turn to the question of quantifying the higher-order terms. The first $\mathscr{O}\left(\alpha_{s}\right)$ correction in (2.3) reads

$$
\mathscr{Q}^{(1)}\left(p_{T}\right)=\int \mathrm{d} z P_{g q}(z) \int \frac{\mathrm{d} \theta}{\theta} \frac{\alpha_{s}}{\pi}\left[\mathscr{Q}_{g q}\left(p_{T}\right)-\mathscr{Q}_{q}\left(p_{T}\right)\right],
$$

where $P_{g q}(z)$ is the Altarelli-Parisi splitting function. The first term describes the real gluon emission and its subsequent quenching, while the second term describes a virtual fluctuation where only the parent quark is affected by energy loss. In the large- $N_{c}$ limit, the quenching of a pair of partons 
is simply the combined effect of the quenching of the total charge, that is related to the color charge of the parent parton, and the additional quenching related to the additional color charge generated in the splitting [15]. In Laplace space we can simply write $\mathscr{Q}_{g q}\left(p_{T}\right)=\mathscr{Q}_{q}\left(p_{T}\right) \mathscr{Q}_{\text {sing }}\left(p_{T}\right)$, and the quark quenching factor that is common in both terms in (2.6) can be factored out. While Eq. (2.6) describes a $\mathscr{O}\left(\alpha_{s}\right)$ correction, it could become sizable in a region of phase space where the quenching affects the color singlet dipole. Let us therefore proceed with a brief discussion of the singlet quenching weight $\mathscr{Q}_{\text {sing }}\left(p_{T}\right) \equiv \tilde{\mathscr{P}}_{\text {sing }}\left(p_{T} / n\right)$.

It was shown [15] that the resummation of multiple induced gluons for the probability of energy loss off a color singlet dipole involves both direct and interference terms. The resulting rate equation reads,

$$
\frac{\partial}{\partial t} \tilde{P}_{\text {sing }}(v, t)=2 \gamma_{\text {dir }}(v, t) \mathscr{P}_{\text {sing }}(v, t)+\gamma_{\text {int }}(v, t) \mathscr{S}_{2}(t) .
$$

In this case, the rate of direct emissions is identical to the one in (2.4), $\gamma_{\mathrm{dir}}(v, t)=\gamma(v, t)$. The interference term is simply $\gamma_{\text {int }}(v, t)=-2 \gamma(v, t)$ for soft gluons, due to color charge conservation. However, the interference term involves a dipole suppression factor describing the survival probability of color coherence at a given time in course of the dipole propagation. It is called the decoherence parameter [10,11], and reads

$$
\mathscr{S}_{2}(t)=\exp \left(-\frac{1}{12} \hat{q} \theta^{2} t^{3}\right)=\exp \left[-\frac{1}{12}\left(\frac{t}{t_{\mathrm{d}}}\right)^{3}\right],
$$

which gives rise to a characteristic time-scale for decoherence $[12,13,14]$. This time-scale can easily be estimated by comparing the medium resolution scale due to multiple scattering $\lambda_{\perp} \sim$ $(\hat{q} t)^{-1 / 2}$ with the size of the dipole $x_{\perp} \sim \theta t$ where $\theta$ is the dipole angle. The two scales become comparable at $t_{\mathrm{d}} \sim\left(\hat{q} \theta^{2}\right)^{-1 / 3}$, which is the so-called decoherence time.

For small angle dipoles, color decoherence takes a long time. In particular, for $t_{\mathrm{d}} \gg L$ or $\theta \gg$ $\theta_{c} \sim\left(\hat{q} L^{3}\right)^{-1 / 2}$, the singlet dipole does not lose energy $\left.\mathscr{Q}_{\text {sing }}\left(p_{T}\right)\right|_{t_{\mathrm{d}} \gg L} \approx 1$. This is a manifestation of color transparency. Hence, logarithmic corrections in (2.6) will only arise as long as $t_{\mathrm{d}} \ll L$, where the singlet quenching factor becomes the product of the independent quenching factors off the dipole constituents, i.e. when $\mathscr{S}_{2} \ll 1$ in (2.7), $\left.\mathscr{Q}_{\text {sing }}\left(p_{T}\right)\right|_{t_{\mathrm{d}} \ll L} \approx \mathscr{Q}_{q}^{2}\left(p_{T}\right)$. Furthermore, we have to demand that the jet is formed sufficiently early in the medium so as not to be interfering with induced emissions, in particular $t_{\mathrm{f}}<t_{\mathrm{d}}$. It can be shown that these considerations capture the leading-logarithmic behavior of the cross section and that a more sophisticated treatment of the phase space leads to sub-leading logarithmic corrections [5].

Returning to Eq. (2.6), it can now be simplified as

$$
\begin{aligned}
\mathscr{Q}^{(1)}\left(p_{T}\right) & \simeq \mathscr{Q}_{q}\left(p_{T}\right) \times 2 \bar{\alpha} \int_{t_{\mathrm{f}}<t_{\mathrm{d}}<L} \frac{\mathrm{d} z}{z} \frac{\mathrm{d} \theta}{\theta}\left[\mathscr{Q}_{q}^{2}\left(p_{T}\right)-1\right], \\
& \simeq \mathscr{Q}_{q}\left(p_{T}\right) \times\left[-2 \bar{\alpha} \log \frac{R}{\theta_{c}}\left(\log \frac{p_{T}}{\omega_{c}}+\frac{2}{3} \log \frac{R}{\theta_{c}}\right)\right],
\end{aligned}
$$

for fixed coupling. In going to the second line, we have focussed on the strong quenching regime, i.e. where $\mathscr{Q}\left(p_{T}\right) \ll 1$, where only the virtual term survives. ${ }^{1}$ As becomes clear from (2.10), the

\footnotetext{
${ }^{1}$ This makes sense formally only as long as $\omega_{c}<p_{T}<n \bar{\alpha}^{2} \omega_{c}$ and, hence, our current estimates are only parametric. For realistic cases, the real term plays an important role [5].
} 
correction is enhanced by large logarithms of the phase space related to the jet scales $p_{T}$ and $R$. In particular, the enhancement is single-logarithmic in jet $p_{T}$ because of the finite resolution angle $\theta_{c}>0$, and in contrast to the toy-model considered in the Introduction due to the difference of relevant phase space.

Remarkably, in the large- $N_{c}$ approximation, all higher order terms in the jet suppression factor are directly proportional to the quenching of the total color charge, in the same way as in (2.10). We can therefore show that

$$
R_{\text {jet }}=\mathscr{Q}_{q}\left(p_{T}\right) \times \mathscr{C}\left(p_{T}, R\right),
$$

which is the main result of our analysis. Here, $\mathscr{C}\left(p_{T}, R\right)$ is a novel "collimator" function [5] that accounts for the quenching of higher-order jet fluctuations due to the mismatch of real and virtual contributions. In the strong quenching regime, where only virtual fluctuations contribute, the resummation of all-orders simply amounts to the exponentiation of the first-order correction in (2.10), leading to

$$
\mathscr{C}\left(p_{T}, R\right) \simeq \exp \left[-2 \bar{\alpha} \log \frac{R}{\theta_{c}}\left(\log \frac{p_{T}}{\omega_{c}}+\frac{2}{3} \log \frac{R}{\theta_{c}}\right)\right] .
$$

While the quenching of the "total charge", or the initial parent quark, in Eq. (2.11) does not depend on the jet scales, the additional Sudakov suppression factor is sensitive to them.

We have also generalized this procedure for finite quenching effects, and devised a general resummation formula based on Eq. (2.9) for the "collimator" function that also goes beyond the leading-logarithmic approximation [5]. This results in a system of coupled, non-linear evolution equation for quark and gluon jets, that reads

$$
\begin{aligned}
\mathscr{C}_{i}\left(1, p_{T}, R\right) & =1+\int_{0}^{1} \mathrm{~d} z \int_{\theta_{c}}^{R} \frac{\mathrm{d} \theta}{\theta} \frac{\alpha_{s}\left(k_{\perp}\right)}{\pi} P_{g i}(z) \Theta\left(t_{\mathrm{d}}-t_{\mathrm{f}}\right) \\
& \times\left[\mathscr{C}_{g}\left(z, p_{T}, \theta\right) \mathscr{C}_{i}\left((1-z), p_{T}, \theta\right) Q_{q}^{2}\left(p_{T}\right)-\mathscr{C}_{i}\left(1, p_{T}, \theta\right)\right],
\end{aligned}
$$

where $i=q, g$ and we have restored the full Altarelli-Parisi splitting functions. Note, that the argument of the running coupling in this equation is related to the transverse momentum of jet splittings. We show the resulting jet suppression factor as the solid line in Fig. 1 for a quark jet with opening angle $R=0.4$ (the shaded band corresponds to varying the opening angle by \pm 0.1 ). In addition, we plot the suppression factor of a single quark (dotted line). Finally, if we neglect color (de)coherence effects and implement the simple model outlined in the introduction (this corresponds to changing the relevant phase space in (2.13)), we obtain a stronger suppression (dotted line), reflecting the stronger (double-logarithmic) dependence on jet scales in this case.

The developments we have described in these proceeding can be employed in a phenomenological analysis of experimental data on jet suppression. More importantly, they describe a general way of extending the analysis of energy loss processes to higher orders and gaining theoretical control of their magnitude in terms of jet and medium scale analysis. They also apply directly to substructure observables, and estimates of quenching of two subjets found by grooming, based directly on the real term in Eq. (2.6), were already presented at the previous edition of this conference series [16]. It is also worth pointing out that a similar analysis of medium scales could affect 


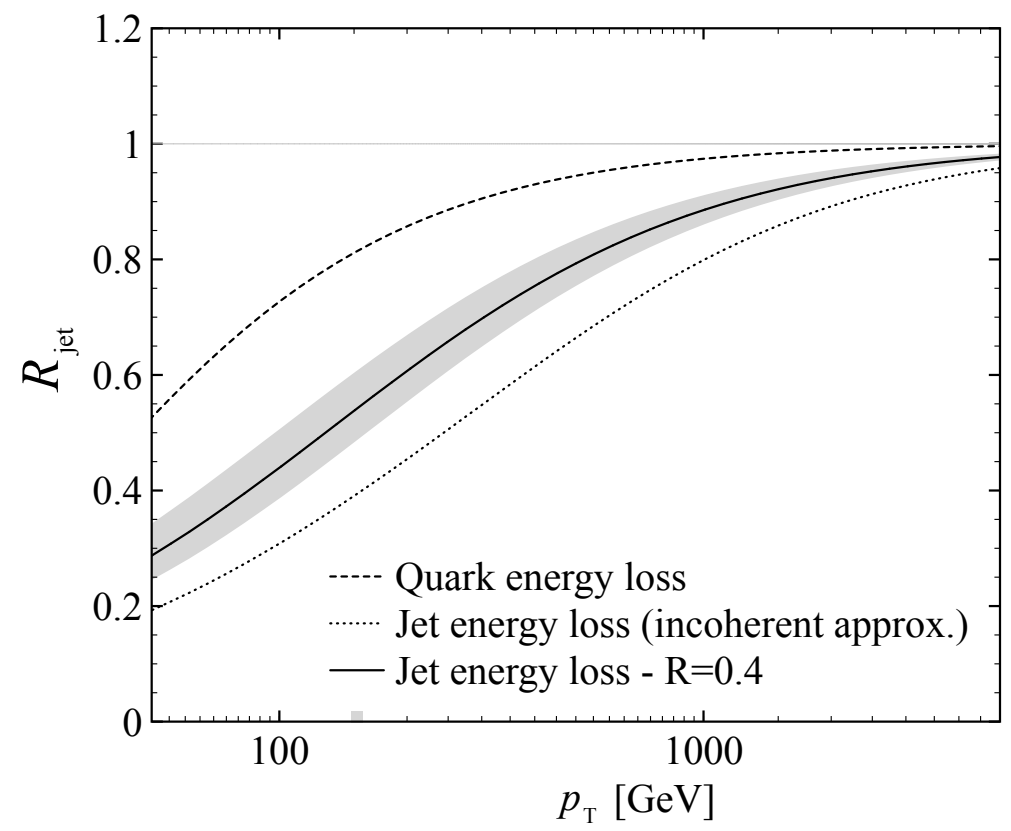

Figure 1: The jet suppression factor for (light) quarks (dashed line), quark jets with coherence effects (solid curve) and without coherence effects (dotted line). Figure taken from [5].

our understanding of low-momentum fragments in jets [17, 18]. Ultimately, these developments will aid in attaining a better theoretical control for Monte-Carlo implementations and will lead to a better grip on the properties of the dense QCD medium created in heavy-ion collisions.

\section{References}

[1] Y. Mehtar-Tani, J. G. Milhano and K. Tywoniuk, Int. J. Mod. Phys. A 28 (2013) 1340013 [arXiv:1302.2579 [hep-ph]].

[2] Y. L. Dokshitzer, V. A. Khoze, A. H. Mueller and S. I. Troian, Gif-sur-Yvette, France: Ed. Frontieres (1991) 274 p. (Basics of)

[3] J. G. Milhano and K. C. Zapp, Eur. Phys. J. C 76 (2016) no.5, 288 [arXiv:1512.08107 [hep-ph]].

[4] J. Casalderrey-Solana, D. Gulhan, G. Milhano, D. Pablos and K. Rajagopal, JHEP 1703 (2017) 135 [arXiv:1609.05842 [hep-ph]].

[5] Y. Mehtar-Tani and K. Tywoniuk, Phys. Rev. D 98 (2018) no.5, 051501 [arXiv:1707.07361 [hep-ph]].

[6] R. Baier, Y. L. Dokshitzer, A. H. Mueller and D. Schiff, JHEP 0109 (2001) 033 [hep-ph/0106347].

[7] R. Baier, Y. L. Dokshitzer, A. H. Mueller, S. Peigne and D. Schiff, Nucl. Phys. B 484 (1997) 265 [hep-ph/9608322].

[8] B. G. Zakharov, JETP Lett. 65 (1997) 615 [hep-ph/9704255].

[9] C. A. Salgado and U. A. Wiedemann, Phys. Rev. D 68 (2003) 014008 [hep-ph/0302184].

[10] Y. Mehtar-Tani, C. A. Salgado and K. Tywoniuk, Phys. Rev. Lett. 106 (2011) 122002 [arXiv:1009.2965 [hep-ph]]. 
[11] Y. Mehtar-Tani, C. A. Salgado and K. Tywoniuk, Phys. Lett. B 707 (2012) 156 [arXiv:1102.4317 [hep-ph]].

[12] J. Casalderrey-Solana and E. Iancu, JHEP 1108 (2011) 015 [arXiv:1105.1760 [hep-ph]].

[13] Y. Mehtar-Tani, C. A. Salgado and K. Tywoniuk, JHEP 1204 (2012) 064 [arXiv:1112.5031 [hep-ph]].

[14] Y. Mehtar-Tani, C. A. Salgado and K. Tywoniuk, JHEP 1210 (2012) 197 [arXiv:1205.5739 [hep-ph]].

[15] Y. Mehtar-Tani and K. Tywoniuk, Nucl. Phys. A 979 (2018) 165 [arXiv:1706.06047 [hep-ph]].

[16] J. Casalderrey-Solana, Y. Mehtar-Tani, C. A. Salgado and K. Tywoniuk, Nucl. Phys. A 967 (2017) 564.

[17] Y. Mehtar-Tani and K. Tywoniuk, Phys. Lett. B 744 (2015) 284 [arXiv:1401.8293 [hep-ph]].

[18] P. Caucal, E. Iancu, A. H. Mueller and G. Soyez, Phys. Rev. Lett. 120 (2018) 232001 [arXiv:1801.09703 [hep-ph]]. 